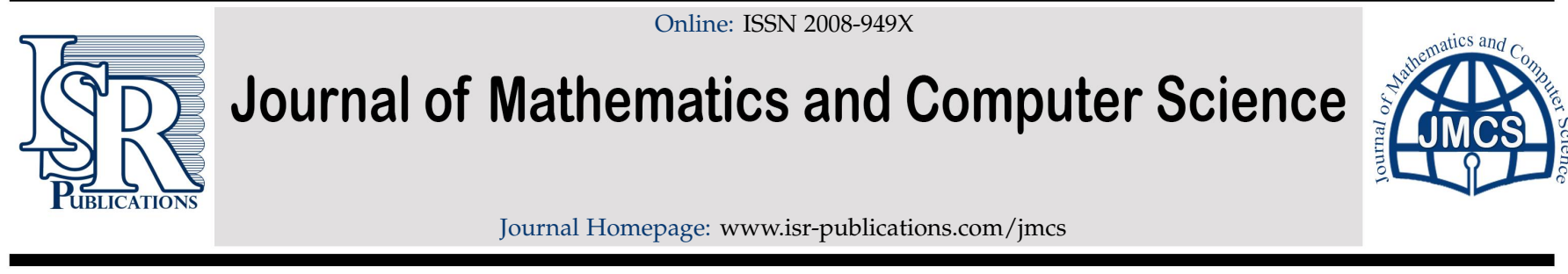

\title{
Distributional chaos in a sequence and topologically weak mixing for nonautonomous discrete dynamical systems
}

\author{
Yu Zhao, Risong Li*, Hongqing Wang, Haihua Liang \\ School of Mathematics and Computer Science, Guangdong Ocean University, Zhanjiang, 524025, P. R. China.
}

\begin{abstract}
Assume that $\left(W, g_{1, \infty}\right)$ is a nonautonomous discrete dynamical system given by sequences $\left(g_{m}\right)_{m=1}^{\infty}$ of continuous maps on the space $(W, d)$. In this paper, it is proven that if $g_{1, \infty}$ is topologically weakly mixing and satisfies that $g_{1}^{n} \circ g_{1}^{m}=g_{1}^{n+m}$ for any $n, m \in\{0,1, \ldots\}$, then it is distributional chaos in a sequence. This result extends the existing one.
\end{abstract}

Keywords: Chaotic in the sense of Devaney, topologically transitive, sensitive, nonautonomous discrete dynamical systems, distributional chaos in a sequence.

2010 MSC: 54H20, 37B40, 37D45.

(C)2020 All rights reserved.

\section{Introduction}

Since Li and Yorke [16] first introduced the term of "chaos" which is known as Li-Yorke chaos today, the dynamical complexity of a dynamical system is a central subject of research. In [3], another definition of chaos, from the viewpoint of topology, was proposed by Devaney. Nonautonomous discrete systems were introduced in [7] (see also [6]). Chaos of nonautonomous discrete dynamical systems has been extensively studied (see [2, 4, 6, 7, 21, 25]). For some related concepts and properties for autonomous discrete dynamical systems we refer the reader to [8-15, 17-19]. A continuous self-map on a metric space is said to be chaotic in the sense of Devaney [1] if it satisfies the following three properties are satisfied: (1) topological transitivity; (2) the denseness of periodic points; (3) sensitive dependence on initial conditions. Similarly, a nonautonomous discrete system is called to be chaotic in the sense of Devaney if the following three properties are satisfied: (1) topological transitivity; (2) the denseness of periodic points; (3) sensitive dependence on initial conditions. The definition of a distributional scrambled set was given for the first time in [24]. In [26], Wang et al. defined distributional chaos in a sequence. Huang and Ye [5] proved that a transitive continuous selfmap from a compact metric space into itself with a fixed point (or a periodic point) is Li-Yorke chaotic. Mai [22] obtained that a scrambled set stronger than that in Li-Yorke's

\footnotetext{
*Corresponding author

Email addresses: datom@189.cn (Yu Zhao), gdoulrs@163.com (Risong Li), wanghq3333@126.com (Hongqing Wang),

lhhlucy@126. com (Haihua Liang)
}

doi: $10.22436 /$ jmcs.020.01.02

Received: 2018-12-14 Revised: 2019-07-13 Accepted: 2019-07-23 
original definition when the transitive system with fixed points is sensitive. In [23], it was showed that Devaney chaotic backward shifts are uniformly distributionally chaotic. In [20] the authors considered the relation between Devaney's chaos and distributional chaos in a sequence for continuous selfmaps on a complete metric space without isolated points. They deduced that if a continuous selfmap on a complete metric space is transitive and has a periodic point of period $p$, then it is distributionally chaotic in a sequence. In particular, for continuous selfmaps on a complete metric space, Devaney's chaos is stronger than distributional chaos in a sequence. In [27], Wang et al. obtained that if a given continuous selfmap $\mathrm{g}: \mathrm{W} \rightarrow \mathrm{W}$ on a separable metric space $\mathrm{W}$ containing at least two points is topologically weakly mixing, then it is distributional chaos in a sequence. In this paper we extend the result of [27] to nonautonomous discrete systems. In particular, it is shown that for a given a nonautonomous discrete dynamical system $\left(W, g_{1, \infty}\right)$ given by sequences $\left(g_{m}\right)_{m=1}^{\infty}$ of continuous maps on the space $W$, if it is topologically weakly mixing and satisfies that $\mathrm{g}_{1}^{\mathfrak{n}} \circ \mathrm{g}_{1}^{\mathrm{m}}=\mathrm{g}_{1}^{\mathrm{n}+\mathrm{m}}$ for any $\mathrm{n}, \mathrm{m} \in\{0,1, \cdots\}$, then it is distributional chaos in a sequence. Thus, our result extends the existing one.

The organization of this paper is as follows. In Section 2, we recall and give some notations and basic concepts. The main results are proved in Section 3.

\section{Preliminaries}

Throughout this paper, we always assume that $(G, \delta)$ is a complete metric space without isolated points, and that $\left(G, h_{1, \infty}\right)$ is a nonautonomous discrete dynamical system given by sequences $\left(h_{j}\right)_{j=1}^{\infty}$ of continuous self-maps $h_{j}: G \rightarrow G$ of the space $G$, where $h_{j}^{m}=f_{m+j-1} \circ \cdots \circ f_{j}$ for every integer $j>0$ and every integer $m \geqslant 0$ and $f_{j}^{0}=i d_{x}$ for every integer $j>0$. For any $C, C^{\prime} \subset G$ with $C, C^{\prime} \neq \emptyset$ and any $s>0$, we write $\delta\left(C, C^{\prime}\right)=\inf \left\{\delta(a, b): a \in C, b \in C^{\prime}\right\}, D(C)=\sup \{\delta(a, b): a, b \in C\}, \delta(a, C)=\inf \{\delta(a, b): b \in C\}$ and $B(C, s)=\{a \in G: \delta(a, C)<\delta\}$. If $C=\{c\}$ then we write $B(c, s)$ (resp. $\delta\left(C, C^{\prime}\right)$ ) for $B(C, s)$ (resp. $\left.\delta\left(c, C^{\prime}\right)\right)$.

A point $w \in G$ is said to be a transitive point of $\left(G, h_{1, \infty}\right)$ or $h_{1, \infty}$ if the orbit

$$
\mathrm{O}\left(w, \mathrm{~h}_{1, \infty}\right)=\left\{\mathrm{h}_{1}^{\mathrm{j}}(w): j \in\{0,1, \cdots\}\right\}
$$

of $w$ under $\left(G, h_{1, \infty}\right)$ or $h_{1, \infty}$ is dense in $G$. It is well known that a nonautonomous discrete dynamical system $\left(G, h_{1, \infty}\right)$ on a compact metric space is transitive if there is a transitive point of $\left(G, h_{1, \infty}\right)$, and that the system $\left(G, h_{1, \infty}\right)$ is transitive if and only if for every pair of nonempty open subsets $S, T \subset G$, there exists an integer $m>0$ such that $h_{1}^{m}(S) \cap T \neq \emptyset$. A nonautonomous discrete dynamical system $\left(G, h_{1, \infty}\right)$ or $h_{1, \infty}$ is said to be minimal if each point of $G$ is a transitive point of $\left(G, h_{1, \infty}\right)$ or $h_{1, \infty}$. A point $u \in G$ is said to be almost periodic if the closure of its orbit under $\left(G, h_{1, \infty}\right)$ or $h_{1, \infty}$ is a minimal subset of $\left(G, h_{1, \infty}\right)$ or $h_{1, \infty}$.

Let $\left\{q_{j}\right\}_{j=1}^{\infty}$ be a sequence of positive integers with $q_{j+1}>q_{j}$ for every integer $j>0$, and let $u, v \in G$ and $t>0$. Write

$$
F_{u v}\left(h_{1, \infty}, t,\left\{q_{j}\right\}_{j=1}^{\infty}\right)=\liminf _{m \rightarrow \infty} \frac{1}{m} \sum_{j=1}^{m} x_{[0, t)}\left(\delta\left(h_{1}^{q_{j}}(u), h_{1}^{q_{j}}(v)\right)\right)
$$

and

$$
\mathrm{F}_{u v}^{*}\left(\mathrm{~h}_{1, \infty}, \mathrm{t},\left\{\mathrm{q}_{j}\right\}_{j=1}^{\infty}\right)=\limsup _{m \rightarrow \infty} \frac{1}{m} \sum_{j=1}^{m} \chi_{[0, t)}\left(\delta\left(h_{1}^{\mathrm{q}_{j}}(\mathrm{u}), \mathrm{h}_{1}^{\mathrm{q}_{j}}(v)\right)\right),
$$

where $\chi_{E}(x)=1$ for any $x \in E$ and $\chi_{E}(x)=0$ for any $x \notin E$.

Let $S \subset \mathrm{G}$ and $u, v \in S$ with $u \neq v$. If the following conditions hold:

(1) $F_{u v}\left(h_{1, \infty}, \alpha,\left\{q_{j}\right\}_{j=1}^{\infty}\right)=0$ for some $\alpha>0$;

(2) $\mathrm{F}_{u v}^{*}\left(\mathrm{~h}_{1, \infty}, \mathrm{t},\left\{\mathrm{q}_{j}\right\}_{j=1}^{\infty}\right)=1$ for any $\mathrm{t}>0$,

then we say that $S$ is a distributively chaotic set of a nonautonomous discrete dynamical system $\left(G, h_{1, \infty}\right)$ or $h_{1, \infty}$ in a sequence, and $u, v$ are said to be a pair of points of a nonautonomous discrete dynamical system $\left(G, h_{1, \infty}\right)$ or $h_{1, \infty}$ displaying distributional chaos in a sequence. 
A nonautonomous discrete dynamical system $\left(G, h_{1, \infty}\right)$ or $h_{1, \infty}$ is called to be distributively chaotic in a sequence if it has a distributively chaotic set in a sequence which is uncountable.

Definition 2.1. A nonautonomous discrete dynamical system $\left(W, g_{1, \infty}\right)$ or $g_{1, \infty}$ is called to be distributional chao in a sequence if there exists a residual set $C \subset W \times W$ (i.e., $C$ contains a countable intersection of everywhere dense sets) satisfying that any $(a, b) \in C \times C$ with $a \neq b$ is a distributively chaotic point pair in a sequence.

Definition 2.2. Let $\left\{p_{j}\right\}_{j=1}^{\infty}$ be an increasing sequence of positive integers and write $\operatorname{PR}\left(g_{1, \infty},\left\{p_{j}\right\}\right)=$ $\left\{(a, b) \in W \times W\right.$ : for any $\varepsilon>0$, there is an integer $j>0$ with $\left.d\left(g_{1}^{\mathfrak{p}_{j}}(a), g_{1}^{p_{j}}(b)\right)<\varepsilon\right\}$. The set $\operatorname{PR}\left(g_{1, \infty},\left\{p_{j}\right\}\right)$ is called to be the proximal relation with respect to $\left\{p_{j}\right\}_{j=1}^{\infty}$ and $\left(W, g_{1, \infty}\right)$.

Definition 2.3. Let $\left\{p_{j}\right\}_{j=1}^{\infty}$ be an increasing sequence of positive integers and write

$$
A R\left(g_{1, \infty},\left\{p_{j}\right\}\right)=\left\{(a, b) \in W \times W: \lim _{j \rightarrow \infty} d\left(g_{1}^{p_{j}}(a), g_{1}^{p_{j}}(b)\right)=0\right\} .
$$

The set $A R\left(g_{1, \infty},\left\{p_{j}\right\}\right)$ is called to be the asymptotic relation with respect to $\left\{p_{j}\right\}_{j=1}^{\infty}$ and $\left(W, g_{1, \infty}\right)$.

Definition 2.4. Let $\left\{p_{j}\right\}_{j=1}^{\infty}$ be an increasing sequence of positive integers and write

$$
\operatorname{DR}\left(g_{1, \infty},\left\{p_{j}\right\}\right)=(W \times W)-\operatorname{PR}\left(g_{1, \infty},\left\{p_{j}\right\}\right) .
$$

The set $\operatorname{DR}\left(g_{1, \infty},\left\{p_{j}\right\}\right)$ is called to be the distal relation with respect to $\left\{p_{j}\right\}_{j=1}^{\infty}$ and $\left(W, g_{1, \infty}\right)$.

Definition 2.5. Let $\left\{p_{j}\right\}_{j=1}^{\infty}$ be an increasing sequence of positive integers and write $\operatorname{DCR}\left(g_{1, \infty},\left\{p_{j}\right\}\right)=$ $\left\{(a, b) \in W \times W:(a, b)\right.$ is a distributively chaotic point pair of $g_{1, \infty}$ in the sequence $\left.\left\{p_{j}\right\}_{j=1}^{\infty}\right\}$. The set $\operatorname{DCR}\left(g_{1, \infty},\left\{p_{j}\right\}\right)$ is called to be the distributively chaotic relation with respect to $\left\{p_{j}\right\}_{j=1}^{\infty}$ and $\left(W, g_{1, \infty}\right)$.

\section{Main results}

The proof of the following theorem is similar to that of Lemma 2 in [28]. For completeness, we give its proof.

Theorem 3.1. Let $\left\{m_{j}\right\}_{j=1}^{\infty} \subset\{1,2, \ldots\}$ and let $\left\{p_{j}\right\}_{j=1}^{\infty} \subset\left\{m_{j}\right\}_{j=1}^{\infty}$ and $\left\{q_{j}\right\}_{j=1}^{\infty} \subset\left\{m_{j}\right\}_{j=1}^{\infty}$ be two infinitely increasing sequences. Then, for any nonautonomous discrete dynamical system $\left(\mathrm{W}, \mathrm{g}_{1, \infty}\right)$ on a separable metric space with at least two points $(\mathrm{W}, \mathrm{d})$, there is an infinitely increasing subsequence $\left\{\mathrm{t}_{\mathbf{j}}\right\}_{\mathbf{j}=1}^{\infty} \subset\left\{\mathrm{m}_{\mathbf{j}}\right\}_{j=1}^{\infty}$ with

$$
\operatorname{AR}\left(g_{1, \infty},\left\{p_{j}\right\}_{j=1}^{\infty}\right) \cap \operatorname{DR}\left(g_{1, \infty},\left\{q_{j}\right\}_{j=1}^{\infty}\right) \subset \operatorname{DCR}\left(g_{1, \infty,}\left\{t_{j}\right\}_{j=1}^{\infty}\right) .
$$

Proof. Write $\left\{s_{j}\right\}_{j=1}^{\infty}=\left\{p_{j}\right\}_{j=1}^{\infty} \cap\left\{r_{j}\right\}_{j=1}^{\infty}$. Clearly,

$$
\operatorname{AR}\left(g_{1, \infty},\left\{p_{j}\right\}_{j=1}^{\infty}\right) \cap \operatorname{DR}\left(g_{1, \infty},\left\{q_{j}\right\}_{j=1}^{\infty}\right) \subset \operatorname{AR}\left(g_{1, \infty},\left\{s_{j}\right\}_{j=1}^{\infty}\right) \cap \operatorname{DR}\left(g_{1, \infty},\left\{s_{j}\right\}_{j=1}^{\infty}\right) .
$$

If $\left\{s_{j}\right\}_{j=1}^{\infty}$ is an infinite set, then

$$
\operatorname{AR}\left(g_{1, \infty},\left\{p_{j}\right\}_{j=1}^{\infty}\right) \cap \operatorname{DR}\left(g_{1, \infty},\left\{q_{j}\right\}_{j=1}^{\infty}\right)=\emptyset \subset \operatorname{DCR}\left(g_{1, \infty},\left\{t_{j}\right\}_{j=1}^{\infty}\right) .
$$

If $\left\{s_{j}\right\}_{j=1}^{\infty}$ is a finite set, then, without loss of generality, one can assume that $\left\{\boldsymbol{s}_{\mathfrak{j}}\right\}_{j=1}^{\infty}=\emptyset$.

It is easily seen that for any

$$
\begin{gathered}
(a, b) \in \operatorname{AR}\left(g_{1, \infty},\left\{p_{j}\right\}_{j=1}^{\infty}\right) \cap \operatorname{DR}\left(g_{1, \infty},\left\{q_{j}\right\}_{j=1}^{\infty}\right), \\
\lim _{j \rightarrow \infty} d\left(g_{1}^{p_{j}}(a), g_{1}^{p_{j}}(b)\right)=0
\end{gathered}
$$


and

$$
\inf \left\{d\left(g_{1}^{q_{j}}(a), g_{1}^{q_{j}}(b)\right): j \in\{1,2, \ldots\}\right\}>0 .
$$

So, one has that for any $\varepsilon>0$, there is an integer $M>0$ such that $d\left(g_{1}^{p_{j}}(a), g_{1}^{p_{j}}(b)\right)<\varepsilon$ for any integer $j>M$, and that there is $\delta>0$ such that $d\left(g_{1}^{q_{j}}(a), g_{1}^{q_{j}}(b)\right)>\delta$ for any integer $j>M$.

Pick

$$
\left\{n_{j}\right\}_{j=1}^{\infty},\left\{k_{j}\right\}_{j=1}^{\infty} \subset\{1,2, \ldots\}
$$

with $n_{1}=1, n_{j+1}=2 j n_{j}$ and $k_{j}=j^{2}$ for any $j \in\{1,2, \ldots\}$. Also, one can choose an infinitely increasing subsequence $\left\{\mathrm{t}_{j}\right\}_{j=1}^{\infty} \subset\left\{\mathrm{m}_{j}\right\}_{j=1}^{\infty}$ such that for any $j \in\{1,2, \ldots\}$,

$$
\left\{t_{j}: n_{k_{j}-1}<i \leqslant n_{k_{j}}\right\} \subset\left\{p_{j}\right\}_{j=1}^{\infty} \text { and }\left\{t_{j}: n_{k_{j}}<i \leqslant n_{k_{j+1}}\right\} \subset\left\{q_{j}\right\}_{j=1}^{\infty} .
$$

Therefore, one has that for any $\varepsilon>0$ and any sufficiently large $j \in\{1,2, \ldots\}$ with $n_{k_{j}-1}<i \leqslant n_{k_{j}}$,

$$
d\left(g_{1}^{t_{i}}(a), g_{1}^{t_{i}}(b)\right)<\varepsilon .
$$

Hence, one can get that

$$
1 \geqslant \frac{1}{n_{k_{j}}} \sum_{l=1}^{n_{k_{j}}} \chi_{[0, \varepsilon)}\left(d\left(g_{1}^{t_{l}}(a), g_{1}^{t_{l}}(b)\right)\right) \geqslant \frac{n_{k_{j}}-n_{k_{j}-1}}{n_{k_{j}}}=1-\frac{1}{2 j^{2}-2} .
$$

This means that

$$
\lim _{j \rightarrow \infty} \frac{1}{n_{k_{j}}} \sum_{l=1}^{n_{k_{j}}} \chi_{[0, \varepsilon)}\left(d\left(g_{1}^{t_{l}}(a), g_{1}^{t_{l}}(b)\right)\right)=1 .
$$

Clearly, for any sufficiently large $i \in\{1,2, \ldots\}$, if $n_{k_{i}}<l \leqslant n_{k_{i+1}}$, then we have that

$$
d\left(g_{1}^{t_{l}}(a), g_{1}^{t_{l}}(b)\right)>\delta
$$

Hence, one can get that

$$
0 \geqslant \frac{1}{n_{k_{i}+1}} \sum_{l=1}^{n_{k_{i}+1}} \chi_{[0, \varepsilon)}\left(d\left(g_{1}^{t_{l}}(a), g_{1}^{t_{l}}(b)\right)\right) \leqslant \frac{n_{k_{i}}}{n_{k_{i}+1}}=\frac{1}{2 j^{2}}
$$

This means that

$$
\lim _{i \rightarrow \infty} \frac{1}{n_{k_{i}+1}} \sum_{l=1}^{n_{k_{i}+1}} \chi_{[0, \varepsilon)}\left(d\left(g_{1}^{t_{l}}(a), g_{1}^{t_{l}}(b)\right)\right)=0 .
$$

Thus, $(a, b) \in \operatorname{DCR}\left(g_{1, \infty},\left\{t_{j}\right\}_{j=1}^{\infty}\right)$.

Motivated by Lemma 2 in [20] and its proof we can extend this lemma to nonautonomous discrete dynamical systems and establish the following result.

Theorem 3.2. Suppose that $\left(\mathrm{W}, \mathrm{g}_{1, \infty}\right)$ is a transitive nonautonomous discrete dynamical system, where $\mathrm{W}$ is a separable metric space with at least two points. If $\mathrm{g}_{1, \infty}$ is topologically weakly mixing and satisfies that $\mathrm{g}_{1}^{\mathrm{n}} \circ \mathrm{g}_{1}^{\mathrm{m}}=$ $\mathrm{g}_{1}^{\mathrm{n}+\mathrm{m}}$ for any $\mathrm{n}, \mathrm{m} \in\{0,1, \ldots\}$, then it is distributional chaos in a sequence.

Proof. By Theorem 3.1, it is enough to show that there is a residual set $S \subset W$ with

$$
\mathrm{S} \times \mathrm{S} \subset \operatorname{AR}\left(\mathrm{g}_{1, \infty},\left\{\mathrm{t}_{\mathbf{j}}\right\}_{j=1}^{\infty}\right) \cap \mathrm{DR}\left(\mathrm{g}_{1, \infty},\left\{\mathrm{t}_{j}\right\}_{j=1}^{\infty}\right) .
$$

Let $p \in W$ be a transitive point of the system $\left(W, g_{1, \infty}\right)$. Then the positive semiorbit

$$
\mathrm{O}_{g_{1, \infty}}^{+}(p)=\left\{x, g_{1}(p), \ldots, g_{1}^{\mathfrak{n}}(p), \ldots\right\}
$$


of $p$ under $g_{1, \infty}$ is dense in $W$, where $g_{i}^{n}=g_{i+n-1} \circ \cdots \circ g_{i}$ and $g_{i}^{0}$ is the identity map. So, for any nonempty open set $\mathrm{U} \subset \mathrm{W}$, the set

$$
\left\{n: g_{1}^{\mathfrak{n}}(b) \in U, n \in\{0,1, \ldots\}\right\}
$$

is not bounded above. Let $a \in W$ and $M>0$, set

$$
S_{a}(M)=\left\{g_{1}^{s}(a): 0 \leqslant s \leqslant M, s \in\{0,1, \ldots\}\right\} .
$$

Assume that $\mathrm{U} \subset \mathrm{W}$ is an open set and that $\mathrm{M}_{0}>0$ is given. In the following, we will prove that there is an integer $M>M_{0}$ such that $g_{1}^{M}(a) \in U$. Let $V=W-S_{a}\left(M_{0}\right)$. If $V \neq \emptyset$, then $V$ is nonempty and open. As $g_{1, \infty}$ is topologically weakly mixing, $g_{1, \infty}$ is topologically transitive. So, there are $v \in V$ and an integer $s_{v} \geqslant 0$ with $g_{1}^{s_{v}}(v) \in U$. If $v \in S_{a}\left(M_{0}\right)$, then there is $s_{0}>M_{0}$ with $v=g_{1}^{s_{0}}(a)$. Set $s=s_{0}+s_{v}$. Then one has that $s>M_{0}$ and

$$
g_{1}^{s}(a)=g_{1}^{s_{0}+s_{v}}(a)=g_{1}^{s_{v}}\left(g_{1}^{s_{0}}(a)\right)=g_{1}^{s_{v}}(v) \in U .
$$

Assume that $V=\emptyset$ and let $b \in W$. Then $b \in O_{g_{1, \infty}}^{+}(a)$. That is, there is $s_{b} \geqslant 0$ with $b=g_{1}^{s_{b}}(a)$. So, there is a periodic positive semiorbit $\mathrm{P}$ with $\mathrm{g}_{1}^{\mathrm{s}}(\mathrm{b}) \in \mathrm{P}$ for some $s \geqslant 0$. Put

$$
\bar{s}=\left\{s \geqslant 0: g_{1}^{s}(b) \in P, s \in\{0,1, \ldots\}\right\}
$$

and

$$
A_{b}=\left\{g_{1}^{s}: s \geqslant \frac{1}{2} \bar{s}, s \in\{0,1, \ldots\}\right. \text {. }
$$

Set $V_{1}=W-S_{a}\left(\frac{1}{2} \bar{s}+s_{b}\right)$ and $V_{2}=W-A_{b}$. If $\bar{s}>0$, then $V_{1} \subset W$ and $V_{2} \subset W$ are nonempty open sets. Then, for any $q \in V_{1}$, there is $s_{q}>\frac{1}{2} \bar{s}$ with $q=g_{1}^{s_{q}}(b)$. As, $s_{q}+s>\frac{1}{2} \bar{s}$ for any integer $s \geqslant 0$,

$$
g_{1}^{s}(q)=g_{1}^{s}\left(g_{1}^{s_{q}}(b)\right)=g_{1}^{s_{q}+s}(b) \in A_{b}
$$

for any integer $s \geqslant 0$. By the definition of $V_{2}$, one can see that $g_{1}^{s}(q) \notin V_{2}$ for any integer $s \geqslant 0$. That is, $g_{1}^{s}\left(V_{1}\right) \cap V_{2}=\emptyset$ for any integer $s \geqslant 0$. This contradicts the transitivity of $g_{1, \infty}$. Consequently, one has that $\bar{s}=0$. That is, $g_{1}^{0}(b)=b \in P$. Obviously, there exists $M>M_{0}$ with $g_{1}^{M}(a)=b \in U$.

Let $S=\left\{(x, y) \in W \times W:\left\{\left(g_{1}^{s}(x), g_{1}^{s}(y)\right): s \in\{0,1, \ldots\}\right\}\right.$ is dense $\}$. As $W$ is a separable metric space, $W \times W$ is a separable metric space. So, $W \times W$ has a countable base $\left\{U_{1}, U_{2}, \ldots\right\}$. It is easily seen that

$$
\bigcap_{\mathrm{m}=1}^{\infty} \bigcup_{\mathrm{s}=0}^{\infty} \mathrm{g}_{1}^{-\mathrm{s}} \times \mathrm{g}_{1}^{-\mathrm{s}}\left(\mathrm{u}_{\mathrm{m}}\right),
$$

where $g_{1}^{-s} \times g_{1}^{-s}\left(U_{m}\right)=\left(g_{1}^{s} \times g_{1}^{s}\right)^{-1}\left(U_{m}\right)$. It is clear that for any integer $m \geqslant 1$,

$$
\bigcup_{s=0}^{\infty} g_{1}^{-s} \times g_{1}^{-s}\left(u_{m}\right) \subset w \times W
$$

is nonempty and open. By the the topologically weak mixing of $g_{1, \infty}$,

$$
\bigcup_{s=0}^{\infty} g_{1}^{-s} \times g_{1}^{-s}\left(u_{m}\right)
$$

is dense. This shows that $S \subset W \times W$ is a residual set. Choose $x_{0}, y_{0} \in W$ with $x_{0} \neq y_{0}$. By the above argument, for any $(x, y) \in S$ there exist increasing sequences $\left\{s_{i}\right\}_{i=}^{\infty} \subset\{1,2, \ldots\}$ and $\left\{s_{i}^{\prime}\right\}_{i=}^{\infty} \subset\{1,2, \ldots\}$ of integers with

$$
\lim _{i \rightarrow+\infty} d\left(g_{1}^{s_{i}}(x), g_{1}^{s_{i}}(y)\right)=d\left(x_{0}, x_{0}\right)=0 \quad \text { and } \quad \lim _{i \rightarrow+\infty} d\left(g_{1}^{s_{i}^{\prime}}(x), g_{1}^{s_{i}^{\prime}}(y)\right)=d\left(x_{0}, y_{0}\right)>0
$$


Therefore,

$$
(x, y) \in \operatorname{AR}\left(g_{1, \infty},\left\{s_{j}\right\}_{j=1}^{\infty}\right) \cap \operatorname{DR}\left(g_{1, \infty},\left\{s_{j}^{\prime}\right\}_{j=1}^{\infty}\right) .
$$

By Theorem 3.1, there is $\left\{\mathbf{t}_{i}\right\}_{i=1}^{\infty} \subset\left\{s_{j}\right\}_{j=1}^{\infty} \cup\left\{s_{j}^{\prime}\right\}_{j=1}^{\infty}$ with

$$
\operatorname{AR}\left(g_{1, \infty},\left\{s_{j}\right\}_{j=1}^{\infty}\right) \cap \operatorname{DR}\left(g_{1, \infty},\left\{s_{j}^{\prime}\right\}_{j=1}^{\infty}\right) \subset \operatorname{DCR}\left(g_{1, \infty},\left\{t_{j}\right\}_{j=1}^{\infty}\right) .
$$

Consequently, $S$ is the set of distributional chaos in the sequence $\left\{t_{i}\right\}_{i=1}^{\infty}$. This means that $g_{1, \infty}$ is distributional chaos in a sequence.

\section{Acknowledgment}

This research was supported the Opening Project of Artificial Intelligence Key Laboratory of Sichuan Province (2018RZJ03) and the Opening Project of Bridge Non-destruction Detecting and Engineering Computing Key Laboratory of Sichuan Province (2018QZJ03).

\section{References}

[1] J. Banks, J. Brooks, G. Cairns, G. Davis, P. Stacey, On Devaney's definition of chaos, Amer. Math. Monthly, 99 (1992), 332-334. 1

[2] J. S. Cánovas, Li-Yorke chaos in a class of nonautonomous discrete systems, J. Differnce Equ. Appl., 17 (2011), $479-486$. 1

[3] R. L. Devaney, An introduction to chaotic dynamical systems, Addison-Wesley Publishing Co., Redwood City, (1989). 1

[4] J. Dvořáková, Chaos in nonautonomous discrete dynamical systems, Commun. Nonlinear Sci. Numer. Simul., 17 (2012), 4649-652. 1

[5] W. Huang, X. D. Ye, Devaney's chaos or 2-scattering implies Li-Yorke's chaos, Topology Appl., 117 (2002), 259-272. 1

[6] S. Kolyada, M. Misiurewicz, L. Snoha, Topological entropy of nonautonomous piecewise monotone dynamical systems on the interval, Fund. Math., 160 (1999), 161-181. 1

[7] S. Kolyada, S. Snoha, Topological entropy of nonautononous dynamical systems, Random Comput. Dyn., 4 (1996), 205-233. 1

[8] R. S. Li, A note on the three versions of distributional chaos, Commun. Nonlinear Sci. Numer. Simul., 16 (2011), 1993-1997.

[9] R. S. Li, A note on shadowing with chain transitivity, Commun. Nonlinear Sci. Numer. Simul., 17 (2012), $2815-2823$. 1

[10] R. S. Li, A note on stronger forms of sensitivity for dynamical systems, Chaos Solitons Fractals, 45 (2012), $753-758$.

[11] R. S. Li, A note on uniform convergence and transitivity, Chaos Solitons Fractals, 45 (2012), 759-764.

[12] R. S. Li, The large deviations theorem and ergodic sensitivity, Commun. Nonlinear Sci. Numer. Simul., 18 (2013), 819-825.

[13] R. S. Li, A note on chaos and the shadowing property, Int. J. Gen. Syst., 45 (2016), 675-688.

[14] R. S. Li, Several sufficient conditions for a map and a semi-flow to be ergodically sensitive, Dyn. Syst., 33 (2018), 348-360.

[15] R. S. Li, T. X. Lu, Chaos in a topologically transitive semi-flow, J. Nonlinear Sci. Appl., 10 (2017), 1675-1682. 1

[16] T.-Y. Li, J. A. Yorke, Period three implies chaos, Amer. Math. Monthly, 82 (1975), 985-992. 1

[17] R. S. Li, Y. Zhao, H. Q. Wang, Furstenberg families and chaos on uniform limit maps, J. Nonlinear Sci. Appl., 10 (2017), 805-816. 1

[18] R. S. Li, Y. Zhao, H. Q. Wang, R. Jiang, H. H. Liang, F-sensitivity and ( $\left.\mathcal{F}_{1}, \mathcal{F}_{2}\right)$-sensitivity between dynamical systems and their induced hyperspace dynamical systems, J. Nonlinear Sci. Appl., 10 (2017), 1640-1651.

[19] R. S. Li, X. L. Zhou, A note on chaos in product maps, Turkish J. Math., 37 (2013), 665-675. 1

[20] H. Liu, L. D. Wang, Z. Y. Chu, Devaney's chaos implies distributional chaos in a sequence, Nonlinear Anal., 71 (2009), 6144-6147. 1, 3

[21] T. X. Lu, G. R. Chen, Proximal and syndetical properties in nonautonomous discrete systems, J. Appl. Anal. Comput., 7 (2017), 92-101. 1

[22] J.-H. Mai, Devaney's chaos implies existence of s-scrambled sets, Proc. Amer. Math. Soc., 132 (2004), 2761-2767. 1

[23] F. Martinez-Giménez, P. Oprocha, A. Peris, Distributional chaos for backward shifts, J. Math. Anal. Appl., 351 (2009), 607-615. 1

[24] B. Schweizer, J. Smital, Measures of chaos and spectral decomposition of dynamical systems of the interval, Trans. Amer. Math. Soc., 344 (1994), 737-754. 1 
[25] X. Tang, G. R. Chen, T. X. Lu, Some Iterative Properties of $\left(\mathcal{F}_{1}, \mathcal{F}_{2}\right)$-Chaos in Non-Autonomous Discrete Systems, Entropy, 2018 (2018), 9 pages. 1

[26] L. D. Wang, G. Huang, S. M. Huan, Distributional chaos in a sequence, Nonlinear Anal., 67 (2007), 2131-2136. 1

[27] L. D. Wang, Y. Yang, Z. Y. Chu, G. F. Liao, Weakly mixing implies distributional chaos in a sequence, Modern Phys. Lett. B, 24 (2010), 1595-1660. 1

[28] R.-S. Yang, Distribution chaos in a sequence and topologically mixing, Acta Math. Sinica (Chin. Ser.), 45 (2002), $752-758$. 3 